\title{
Energy Simulation for the Integration of Virtual Power Plants into Production Planning
}

\author{
Volker Stich, Ulrich Brandenburg, and Julian Krenge \\ Institute for Industrial Management at RWTH Aachen University, \\ Campus-Boulevard 55, 52074 Aachen, Germany \\ \{Volker.Stich, Ulrich.Brandenburg, \\ Julian.Krenge\} afir.rwth-aachen.de
}

\begin{abstract}
Germany pushes its energy industry towards renewable energy sources which results in an increase of distributed energy generation. Therefore, energy supply will fluctuate more and more and companies need to react to higher and volatile prices as well as to a fluctuating supply of energy. One reaction is to reactively consider these changing prices and supply of energy within production planning and control (PPC). PPC therefore acts as a control unit to integrate volatile energy supply into the production process. Within this paper a scenario is considered where a virtual power plant (VPP) combines different types of distributed energy generation. Energy simulation is then used to demonstrate how fluctuating energy prices can be considered within PPC.
\end{abstract}

Keywords: Energy-Efficiency, Energy Simulation, Production Planning and Scheduling, Virtual Power Plants.

\section{Introduction}

The results of the climate change and the global warming are becoming more visible every day [1] [2]. Inefficient electricity energy consumption accounts for $80 \%$ of all greenhouse gases (GHG) emission in the EU. As a result, the EU has been committed to a new plan (known as 20/20/20) to fight global warming. According to the plan, by 2020 the EU will have cut greenhouse gas emissions by $20 \%$ from 1990 levels, increased renewable energy usage by $20 \%$, and cut energy consumption through improved energy efficiency by $20 \%$ [3].

As Europe shifts away from fossil fuels, electricity will become an even more important energy sector, and more than 29 European countries have targets for a share of renewables of 5-33\% until 2020. Achieving these goals is vital for the EU internal energy market. The implementation of more intelligent and active transmission, distribution and supply systems in the form of Smart Grids is central to the success of such a development. Smart Grids are essential for the integration of distributed energy generation in the power grid, which results in a number of benefits from a sustainability point of view [4]. 
On the downside, along with the increasing share of renewable energies in the electricity network, the supply uncertainty and the volatility increase. One possibility to react to this situation is called demand-side-integration which focuses on increasing the demand flexibility of energy consumers. As the manufacturing industry is one of the major consumers of energy there is a need for companies to play a vital role in this transition process. This can be done by proactively integrating fluctuating energy sources such as wind, solar and water into production planning [5].

\section{Terminology and Scope of the Study}

\subsection{Terminology}

Production logistics focuses on ensuring a better delivery capability and reliability while minimizing logistics and production costs. Therefore, production logistics improves the productivity in a network by organizing material flow through the producing process. Because production logistics strongly influences the important performance indicators delivery capability and delivery reliability, much effort is taken in industrial application and scientific research on this topic [6].

Production planning and control (PPC) is a key function to every producing company. As a value adding part of the company's organization, the main task is the holistic optimization of the production system. Part of this is the (mathematical) optimization of the production program, the material requirements and the production order [7].

Virtual Power Plants (VPP) unite different types of energy generating units, collectively controlled in order to behave like a conventional power plant. Through the use of various energy generation technologies, a continuous, controllable power generation is enabled as it would not be possible at individual power plants working with renewable energy. Connected are for example photovoltaic, wind and hydro power plants. The usage of Virtual Power Plants is an attempt to use the benefit of the usage of renewable energy but still minimize the fluctuations in the power supply [8].

Simulation is the method of evaluating the performance of an existing or proposed system in order to simulate its behavior under different configurations [9]. Since energy costs will rise continuously, optimizing the energy consumption becomes an interesting opportunity to reduce costs. Simulation can be broadly divided as Mathematical methods and Numerical computer-based simulation. Mathematical models involve the use of various theories for reaching a conclusion. They use a few number of parameters but can reach a very precise result. The draw-back of this system is that it sometimes may prove to be very tedious and almost impossible for complex systems. Therefore for solving complex systems and to provide simple solutions, numerical computer-based simulations are used [9]. More specifically, within Energy Simulation the energy flow and consumption is simulated taking into account the material flow process. To optimize the energy efficiency of a production system or parts of the system, the results of these simulations may be used e.g. to determine the optimum batch size and the production sequence. [10] 


\subsection{Scope of the Study}

The study concentrates on discrete manufacturing systems (e.g. Automotive and mechanical engineering). Products to be considered are multi-part products comprising of a simple structure that are being manufactured in small to medium batch-sizes. There is more than one value-added step to the product meaning that each product passes at more than one value-adding element within the production system. The production system is organized as a job shop production therefore machines of the same production technology (e.g. milling, drilling etc.) are set in the same area. The study solely focuses on the area of production excluding interactions with technical building systems (heating, air-conditioning etc.). Additionally, the study focuses on a shortterm examination (weeks) therefore factory layout planning etc. is not considered. As type of energy, solely electrical energy is considered as this type of energy is provided by VPPs.

\section{State-of-the-Art}

In order to include the energy and resource efficiency as a target value into production planning systems, it is necessary to know and understand which factors can influence efficiency and how sophisticated production planning can improve it. Several works have been published in the field of energy consumption of production machines.

The first of these approaches, which has been used by several authors is to analyze the energy consumption of machine tools using high resolution power measurements and assigning specific loads to each operational state of the machine [11], [12], [13].

Weinert et. al. introduced a methodology for planning and operating energy efficient production systems. The key element of this methodology is the knowledge of the power consumption profile of individual machines and the subsequent approximation of the production systems' power consumption using EnergyBlocks. The power consumption profile for the machines used in Weinert's method does not explicitly differentiate the subsystems of a production machine but it represents a machine specific consumption profile. The power consumption profile is approximated by EnergyBlocks and divided in time-variable, production dependent elements and nonvariable elements. All elements are analyzed and the dependence on each other is described [13].

Abele et.al. present a simulation-based method for the prediction of the energy consumption of a running machine [14]. A key finding of their approach is that $43 \%$ of the specific energy consumption results from the base load of the machine. Therefore, the production process is not very significant for the prediction of the total energy consumption. Once again the machine's base load and dynamic power components are identified as the main drivers behind the energy consumption. Their determination is a key element in assessing the consumption of a machine tool.

Schrems et. al. propose a methodology for designing an energy and resource efficient process chain. A decision-based model was developed in order to identify the preferred production alternative out of several possibilities. The method implements a multi-criteria decision system, weighing ecological, technological and economical parameters to generate the result [15]. 


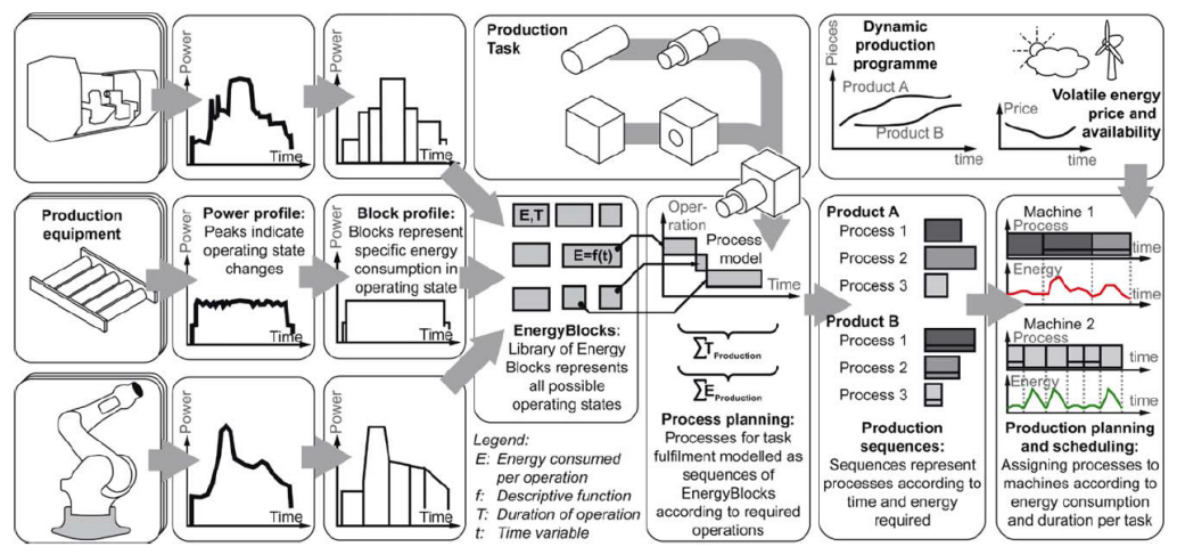

Fig. 1. Energy Blocks method according to [13]

Bruzzone et. al. [16] also propose a mathematical model. This model integrates energy-aware scheduling (EAS) through an advanced planning and scheduling (APS) system. Key to Bruzzone's proposal is an incorporated model that controls the power peaks for a given schedule on the shop floor level. The EAS operates sequentially with the APS. Both share the same optimization criteria and their output is a feasible schedule regarding the main objectives: job-completion-time, cycle time and delayminimization, additionally the power peak target has been added as a criterion.

Pechman and Schöler [17] present an E-PPC system that is able to create a $24 \mathrm{~h}$ energy consumption forecast using a similar technique as proposed by Weinert [13]. This technique allows the user to identify upcoming energy peaks in the phase of production planning, therefore allowing him to reschedule accordingly. With a detailed consumption forecast the company has benefits when negotiating the supply [13] contract with the power utility.

\section{$4 \quad$ Energy Simulation}

The main objective of the energy simulation is to demonstrate how volatile energy sources represented by a VPP can be integrated into producing company without compromising the productivity of the production system. The software used for this study is called PlantSimulation by Siemens PLM Software which contains an Energy Analyzer module. Therewith, it is possible to model machine profiles according to the EnergyBlocks methodology proposed by Weinert [13], [18]. Fig. 2 illustrates the system under study defined in the scope in 2.2. The modelling of the system is done in accordance to März and Weigert and consists of a model of the production system, actuating variables within PPC, input data and target values [19]. 


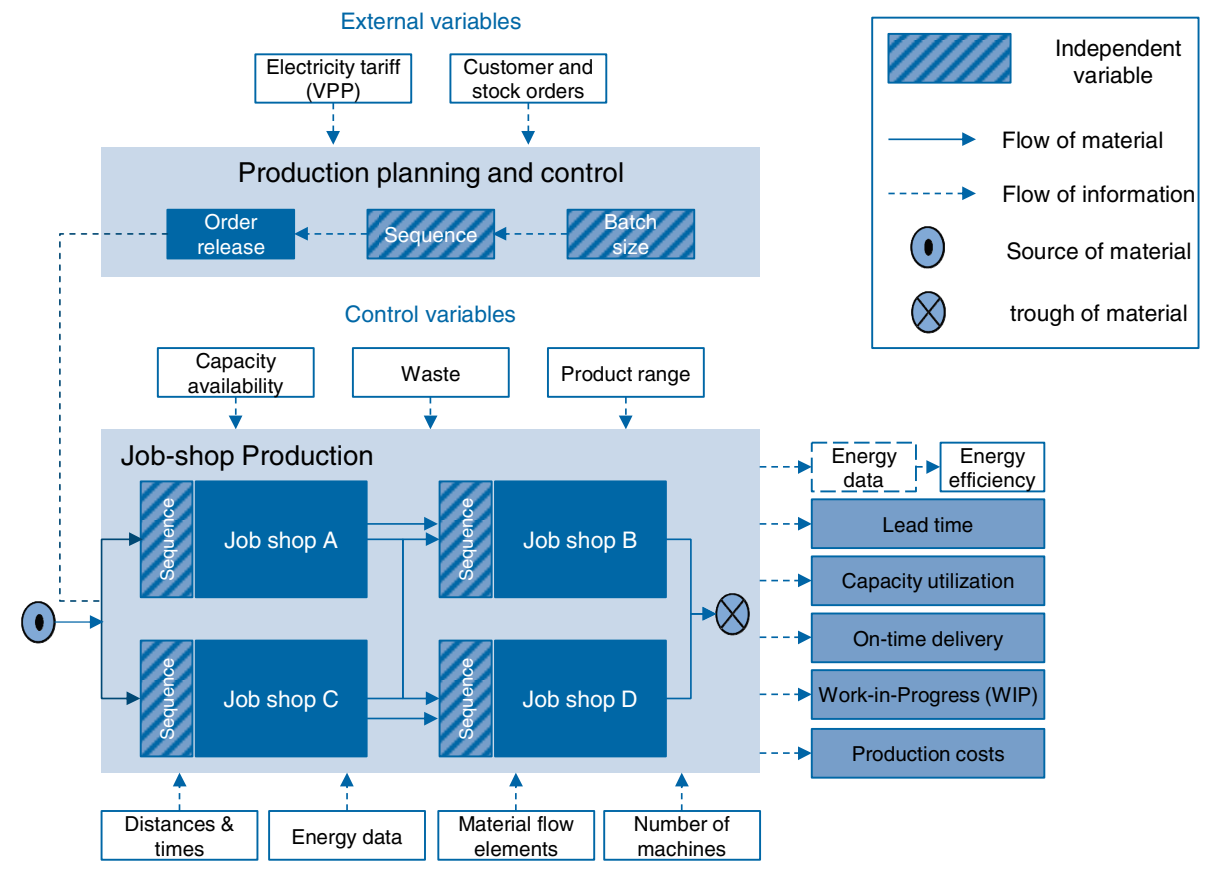

Fig. 2. Energy Simulation model for integrating VPP

The target values have been derived from production logistics such as lead times, capacity utilization and production costs while integrating a target value for energy efficiency. The following target values $Z_{i n t, d y n}$ are defined:

Table 1. Target values within Energy Simulation

\begin{tabular}{|l|c|}
\hline \multicolumn{1}{|c|}{ Name } & Definition \\
\hline Energy Efficiency & $\frac{\sum \text { Energy Costs per part }}{\sum \text { Value }- \text { added per part }}$ \\
\hline Capacity Utilization & $\frac{\sum \text { Time of capacity usage }}{\text { Duration of simulation }}$ \\
\hline Lead time & $T_{i}=$ running time of the simulation \\
& $\frac{T_{\text {output }}-T_{\text {input }} \text { with }}{\text { at } \text { point } \text { in } i}$ \\
\hline On-time delivery & $\frac{\sum \text { on }- \text { time deliveries }}{\sum \text { deliveries }}$ \\
\hline
\end{tabular}


Table 1. (continued)

\begin{tabular}{|l|c|}
\hline Production costs & $\sum$ energy costs, machining costs \\
\hline $\begin{array}{l}\text { Work-in-progress } \\
\text { (WIP) }\end{array}$ & $\frac{\sum_{i=1}^{n} \text { stock }_{i}}{n}$ with \\
& $\begin{array}{l}i=\text { point in time of sampling } \\
n=\text { simulation time in hours }\end{array}$ \\
\hline
\end{tabular}

According to the efficiency definition energy efficiency is considered as a ratio between input and output. In order to account for fluctuating energy prices the input ration is defined as the energy costs that arise per part. The output can be defined as the value-added per part which can be simplified by production costs.

VPP are represented by a volatile energy price function that accounts for the fluctuating feed in of renewable energy sources. Within production planning orders from different sources (customers etc.) are transformed into production orders. For those production orders lot sizes and production sequences are planned based on energy prices and availability, delivery dates etc. The simulation period is considered to be 4 weeks with a weekly interval of releasing production orders into the job shop. Within the job shop a detailed sequencing for each production capacity (machines or groups of machines) is conducted. Therefore, energy prices are considered to be changing every hour. Additionally, energy prices are considered to be known in advance for the following day of production. Within the simulation model elements of the production system are modelled such as machines, buffers and transportation systems. For each element in the system energy data in different states can be defined in order to obtain the energy consumption profiles. According to the principle of discrete-event simulation the material flow pattern generates different operational states of the model such as producing, waiting, failure, setup, standby, etc. according to the arrival rate of products. After conducting the simulation runs, the output data is analyzed. Based on the parameter settings for lot-sizes, production sequence and order release all of the target values are calculated. Then feasible production plans are selected and communicated towards the operator of the VPP.

\section{Conclusion and Outlook}

Rising and volatile energy prices due to an increase of renewable energy sources causes producing companies to strive for integrating VPPs into their production. Within this paper an approach for integrating volatile energy sources into production planning and control was presented. First results of the simulation run are promising that integrating volatile energy prices without compromising productivity can be achieved. The next step will be to implement the results within a real system and validate the results from the energy simulation. Consequently, the results will be used to develop a concept for collaborative production planning within a VPP network. Based on the results from the real system the energy simulation can be used to forecast the 
energy consumption for a given production plan. This information can then be shared with the VPP provider. The first results suggest that rough planning in production (weeks/months) can be used to secure on-time delivery for critical orders due to its high-priority for the customers of producing companies. Once a production plan is validated and the energy consumption is forecasted and communicated to the VPP operator the detailed planning has to ensure that the energy forecast will be realized. Therefore, production sequencing will be very crucial for achieving a high energyefficiency on the shop-floor level. Once the new concepts for energy-efficient production planning are validated within a real production system consequently EnterpriseRessource-Planning-systems and Manufacturing Execution Systems (MES) have to be adapted in order to support energy-efficient production planning.

Acknowledgement. The authors wish to thank the Finesce Consortium for their input and their support. The Finesce project is funded by the EU grant FP7-2012-ICT-FI.

\section{References}

[1] Kara, S., Li, W.: Unit process energy consumption models for material removal processes. CIRP Annals - Manufacturing Technology 60(1), 37-40 (2011)

[2] Branker, K., Jeswiet, J., Kim, I.Y.: Greenhouse gases emitted in manufacturing a product-A new economic model. CIRP Annals - Manufacturing Technology 60(1), 53-56 (2011)

[3] European Commission: 2020 vision. Saving our energy. Luxembourg (2007)

[4] Diaz-Elsayed, N., Jondral, A., Greinacher, S., Dornfeld, D., Lanza, G.: Assessment of lean and green strategies by simulation of manufacturing systems in discrete production environments. CIRP Annals - Manufacturing Technology 62(1), 475-478 (2013)

[5] Reinhart, G., Reinhardt, S., Graß1, M.: Energieflexible Produktionssysteme. Einführungen zur Bewertung der Energieeffizienz von Produktionssystemen. wt Werkstattstechnik online 102(9), 622-628 (2012)

[6] Nyhuis, P., Wiendahl, H.-P.: Fundamentals of production logistics. Theory, tools and applications; with 6 tables (CD-ROM included), Berlin, Heidelberg (2009)

[7] Schuh, G., Stich, V.(Hrsg.): Grundlagen der PPS. Springer, Berlin (2012)

[8] Fang, X., Misra, S., Xue, G., Yang, D.: Smart Grid - The New and Improved Power Grid: A Survey. IEEE Communications Surveys \& Tutorials 14(4), 944-980 (2012)

[9] Banks, J., Carson II, J.S., Nelson, B.L., Nicol, D.M.: Discrete-event system simulation, 5. Auflage, Upper Saddle River (2010)

[10] Bangsow, S.(Hrsg.): Use cases of discrete event simulation. Appliance and research, Berlin, New York (2012)

[11] Duflou, J.R., Kellens, K., Renaldi; Guo, Y., Dewulf, W.: Critical comparison of methods to determine the energy input for discrete manufacturing processes. CIRP Annals - Manufacturing Technology 61(1), 63-66 (2012)

[12] Li, W., Winter, M., Kara, S., Herrmann, C.: Eco-efficiency of manufacturing processes: A grinding case. CIRP Annals - Manufacturing Technology 61(1), 59-62 (2012)

[13] Weinert, N., Chiotellis, S., Seliger, G.: Methodology for planning and operating energyefficient production systems. CIRP Annals - Manufacturing Technology 60(1), 41-44 (2011) 
[14] Abele, E., Schrems, S., Schraml, P.: Energieeffizienz in der Fertigungsplanung. Frühzeitige Abschätzung des Energieverbrauchs von Produktionsmaschinen in der Mittel- und Großserienfertigung. wt Werkstattstechnik online 102(1/2), 38 (2012)

[15] Schrems, S., Eisele, C., Abele, E.: Methodology for an Energy and Resource Efficient Process Chain Design. In: Hesselbach, J., Herrmann, C. (Hrsg.) Glocalized Solutions for Sustainability in Manufacturing, Berlin, Heidelberg (2011)

[16] Bruzzone, A.A.G., Anghinolfi, D., Paolucci, M., Tonelli, F.: Energy-aware scheduling for improving manufacturing process sustainability: A mathematical model for flexible flow shops. CIRP Annals - Manufacturing Technology 61(1), 459-462 (2012)

[17] Pechmann, A., Schöler, I.: Optimizing Energy Costs by Intelligent Production Scheduling. In: Hesselbach, J., Herrmann, C. (Hrsg.) Glocalized Solutions for Sustainability in Manufacturing, Berlin, Heidelberg (2011)

[18] Wolff, D., Kulus, D., Dreher, S.: Simulating Energy Consumption in Automotive Industries. In: Bangsow, S. (Hrsg.) Use cases of discrete event simulation. Appliance and Research, Berlin (2012)

[19] März, L., Weigert, G.: Simulationsgestützte Optimierung. In: März, L., Krug, W., Rose, O., Weigert, G. (Hrsg.) Simulation und Optimierung in Produktion und Logistik, Berlin, Heidelberg (2011) 